\title{
Antibodies to Plasmodium falciparum merozoite surface protein-1 p19 malaria vaccine candidate induce antibody-dependent respiratory burst in human neutrophils
}

\author{
Charlotte Joos ${ }^{1,2+}$, Marie-Louise Varela ${ }^{1 \dagger}$, Babacar Mbengue ${ }^{3}$, Annick Mansourou', Laurence Marrama ${ }^{4}$, \\ Cheikh Sokhna ${ }^{5}$, Adama Tall ${ }^{4}$, Jean-François Trape ${ }^{5}$, Aissatou Touré ${ }^{1}$, Odile Mercereau-Puijalon² \\ and Ronald Perraut ${ }^{*}$
}

\begin{abstract}
Background: Identification of plasmodial antigens targeted by protective immune mechanisms is important for malaria vaccine development. Among functional assays, the neutrophil antibody-dependent respiratory burst (ADRB) induced by opsonized Plasmodium falciparum merozoites has been correlated with acquired immunity to clinical malaria in endemic areas, but the target merozoite antigens are unknown. Here, the contribution of antibodies to the conserved C-terminal domain of the P. falciparum merozoite surface protein-1 (PFMSP1 1919) in mediating ADRB was investigated in sera from individuals living in two Senegalese villages with differing malaria endemicity.

Methods: Anti-PfMSP1 1919 antibody levels in sera from 233 villagers were investigated and the involvement of antiPfMSP1 19 antibodies in ADRB was explored in a subset of samples using (1) isogenic $P$. falciparum parasite clones expressing P. falciparum or Plasmodium chabaudi MSP1 p19; (2) PfMSP1 1 19-coated plaque ADRB; and, (3) ADRB triggering using sera depleted from PfMSP1 19 antibodies by absorption onto the baculovirus recombinant antigen.

Results: ADRB activity correlated with anti-PfMSP1p19 lgG levels $\left(P<10^{-3}\right)$. A substantial contribution of PfMSP1 p19 antibody responses to ADRB was confirmed $\left(P<10^{-4}\right)$ in an age-adjusted linear regression model. PfMSP1 19 antibodies accounted for $33.1 \%$ (range $7-54 \%$ ) and $33.2 \%$ (range $0-70 \%$ ) of ADRB activity evaluated using isogenic merozoites $\left(P<10^{-3}\right)$ and depleted sera $(P=0.0017)$, respectively. Coating of PfMSP1 19 on plates induced strong ADRB in anti-PFMSP1p19-positive sera.
\end{abstract}

Conclusion: These data show that naturally acquired P. falciparum MSP1 p19 antibodies are potent inducers of neutrophil ADRB and support the development of PFMSP1 p19-based malaria vaccine using ADRB assay as a functional surrogate for protection.

Keywords: Malaria, Plasmodium falciparum, ELISA, IgG, Functional assay, Chemiluminescence, Neutrophils, ADRB, MSP1p19, Senegal

\section{Background}

Plasmodium spp. parasites responsible for malaria remain a major global health burden and efforts are being

\footnotetext{
*Correspondence: perraut@pasteur.sn

${ }^{\dagger}$ Charlotte Joos and Marie-Louise Varela contributed equally to this work

${ }^{1}$ Unité d'Immunologie, Institut Pasteur de Dakar, Dakar, Senegal

Full list of author information is available at the end of the article
}

intensified to develop effective vaccines and new therapy. Plasmodium falciparum, which causes about 600,000 deaths each year [1], is becoming multi-drug resistant [2, 3], exacerbating the need for an effective malaria vaccine. Merozoite surface proteins (MSPs) are attractive candidate antigens for vaccine development and several current vaccine candidates are recombinant MSP analogues $[4,5]$. MSPs are expressed by mature intrahepatic forms 
and as such, are possible targets of cellular effectors. MSPs displayed onto the surface of invasive merozoites are directly accessible to host immune effectors in the blood, such as antibodies, complement, neutrophils, or monocytes [6]. Antibodies against various recombinant MSPs have been associated with protection against clinical episodes of $P$. falciparum malaria in endemic settings [7-9]. The exact function of such antibodies is still poorly understood. Merozoite-specific neutralizing antibodies preventing invasion of red blood cells in human sera were evidenced using invasion assays or growth inhibition assays (GIA), but no clear correlation with protection against malaria morbidity has been documented $[9,10]$.

There is an increasing body of evidence to associate protection with presence of cytophilic antibodies [11-13]. Antibody-dependent cellular inhibition (ADCI), whereby monocytes activated by antibodycoated merozoites inhibit development of intracellular erythocytic stages has been associated with protection in humans [14]. Salmon et al. [15] and Kumaratilake et al. [16] showed that merozoite-specific antibodies can induce respiratory bursts from neutrophils (ADRB). The mechanism(s) by which antibody triggered ADRB was then investigated in more detail using recombinant antibodies by Pleass et al., who demonstrated the implication of cytophilic antibodies in ADRB [17, 18]. The respiratory burst activity of human polymorphonuclear neutrophils (PMN) triggered by P. falciparum merozoites and immune IgG from individuals living in endemic areas has been previously characterized and quantified [19]. Importantly, the antibody-dependent respiratory burst (ADRB) activity significantly correlated with acquired clinical protection, suggesting that the release of extracellular oxygen radicals by activated PMNs may represent a key effector mechanism of naturally acquired immunity to malaria [19].

The identification of the merozoite surface antigens reacting with opsonizing antibodies and with antibodies triggering neutrophil activation is of obvious interest for vaccine development as recently investigated in a Plasmodium yoelii malaria mouse model [20].

Here, the role of human antibodies recognizing the C-terminal domain of MSP1, PfMSP1p19, in mediating neutrophil ADRB was investigated. Firstly, the correlation between presence of antibodies to the baculovirusexpressed PfMSP1p19 and ADRB-inducing capacity in the sera from people living in endemic areas was analysed. Secondly, the functional contribution of PfMSP1p19-specific antibodies to ADRB was investigated using P. falciparum D10 wild type or transgenic D10 merozoites, expressing the Plasmodium chabaudi MSP1p19 orthologue [21]. A direct role for PfMSP1p19-specific antibodies in ADRB was further assessed using sera depleted from specific antibodies by affinity chromatography and solid phase ADRB using PfMSP1p19-coated plates [20, 22]. Results show that PfMSP1p19-specific antibodies account for a good proportion of ADRB activity, providing further support for the development of malaria vaccines including the PfMSP1p19 antigen.

\section{Methods}

\section{Study sites, subjects and ethics statement}

This study is part of a longitudinal study conducted in Dielmo and Ndiop, two Senegalese villages with perennial and seasonal transmission, respectively. The sites, population endemicity and the longitudinal surveys carried out have been described previously [23, 24]. In July 2002, 119 Dielmo and 114 Ndiop villagers were enrolled in a longitudinal and cross-sectional study. At the time of recruitment, no villagers were symptomatic for malaria. The mean age of the Ndiop and Dielmo cohorts was 25.3 years (range 3.4-80.5) and 21.8 years (range 3.976.9), respectively; the distribution in the different age groups is shown in Table 1. Blood samples were collected by venous puncture, and sera were stored at $-20^{\circ} \mathrm{C}$.

The project protocol and objectives were carefully explained to the assembled villagers, and informed consent, annually renewed, was obtained individually from all subjects either by signature or by thumbprint on a voluntary consent form written in both French and in the local language (Wolof and Serere) [24]. This study was examined and approved by the Senegalese National Health Research Ethics Committee.

Urban samples were from hospitalized adults with confirmed severe malaria, living in the unstable hypoendemic urban area of Dakar. They were treated at Hôpital Principal, Dakar. Samples were collected day 0 of hospitalization after their use for routine biological investigations. This study was approved by ad hoc Ethics Committee and informed consent was obtained from all participants.

\section{Antigens and antibodies}

The soluble recombinant protein corresponding to PfMSP1p19 was produced in the baculovirus/insect cell expression system in High Five (Invitrogen) insect cells. The construct has a C-terminal hexa-histidine tag that replaces the GPI-modification signal sequence of the parasite protein. Recombinant PfMSP1p19 was purified by metallo-affinity chromatography, as described previously [25].

A hyper-immune serum pool (HIS) from 30 immune, primarily adult residents of Dielmo (mean age 36 years, range 9-73 years), and non-immune serum pool (NIS) obtained commercially (Calbiotech, France), were the positive and negative controls, respectively. 
Table 1 Antibody responses against MSP1p19 in Dielmo and Ndiop villagers tested for ADRB

\begin{tabular}{|c|c|c|c|c|c|c|}
\hline Age groups (years) & $\mathbf{N}$ & Mean age (years) & Anti-PfMSP1p19 levelsa & $\%$ Positive responders $^{\mathbf{b}}$ & $\%$ High responders ${ }^{b}$ & ADRB $^{c}$ \\
\hline \multicolumn{7}{|l|}{ DIELMO } \\
\hline $0-6$ & 19 & 4.6 & $3.6[1-9.1]$ & 68 & 16 & $154[46-354]$ \\
\hline $7-14$ & 31 & 10.9 & $3.5[1-15]$ & 48 & 13 & 231 [40-958] \\
\hline$\geq 15$ & 69 & 37.4 & $9.2[1-16.9]$ & 87 & 62 & 380 [106-575] \\
\hline All & 119 & 25.3 & $6.8[1-16.9]$ & 74 & 42 & 305 [40-958] \\
\hline \multicolumn{7}{|l|}{ NDIOP } \\
\hline $0-14$ & 49 & 8.8 & $6.4[1-18.4]$ & 80 & 37 & $162[51-545]$ \\
\hline $15-29$ & 33 & 20.5 & $9.2[1-19.7]$ & 88 & 55 & $307[113-1721]$ \\
\hline$\geq 30$ & 32 & 43.9 & $11.9[1-19.9]$ & 94 & 69 & 370 [56-1147] \\
\hline All & 114 & 21.8 & 8.8 [1-19.9] & 86 & 51 & $262[51-1721]$ \\
\hline
\end{tabular}

a Mean [range] antibody levels to PfMSP1p19 expressed in OD-ratio

b Percent of responders (OD-ratio > 2) and of High responders (OD-ratio > 7)

c Mean level and range [min-max] of ADRB measured in villagers' sera

\section{ELISA analysis}

The ELISA protocol used to measure PfMSP1p19 antibodies at a 1:200 serum dilution was essentially as described [9, 26, 27], using baculovirus PfMSP1p19 coated on Immulon-4 plates (Dynatech) at $0.5 \mu \mathrm{g} \mathrm{mL}{ }^{-1}$. For inter-assay comparisons, results were expressed as OD-ratios corresponding to OD-sample/OD-naïve. Positive responders (PR) were individuals with an OD-ratio over 2, corresponding to the mean OD of naïve controls $+2 \mathrm{SD}$. High responders (HR) were individuals with an OD-ratio $>7$, i.e. the threshold level previously shown to be significantly associated with anti-parasite activity in re-infection study in Ndiop [26].

To monitor specific antibody depletions quantitatively, samples were analysed at 1:200, 1:400 and 1:800 dilutions, and an arbitrary titre was extrapolated using a four-parameter logistic fit from a standardized positive control regression curve on each plate, determined using the HIS pool and serial twofold dilutions starting at 1:200 $[28,29]$.

\section{Serum depletion}

Sera from high responder individuals to PfMSP1p19 were selected for the depletion studies. Each serum $(100 \mu \mathrm{L})$ was diluted 1:3 in PBS and incubated with $50 \mu \mathrm{g}$ of recombinant hexa-histidine tagged PfMSP1p19 protein for 30 min at RT to allow antigen-antibody binding. Packed TALON Metal Affinity Resin (Ozyme) pre-equilibrated with PBS $(200 \mu \mathrm{L})$, was added and incubated with gentle mixing for $3 \mathrm{~h}$ at room temperature (RT), to allow antigen-antibody complex binding via the $\mathrm{C}$-terminal hexa-histidine tag. Depleted sera were recovered in the supernatant after centrifugation without further dilution, so that initial and depleted sera were directly comparable. Effective depletion was checked by ELISA.

\section{Parasite culture and merozoite preparation}

Plasmodium falciparum parasites (PAM, an FCR3-like background) and P. falciparum D10 (D10-PfM3') or transgenic D10 merozoites, in which PfMSP1p19 is replaced by the non-cross reactive Plasmodium chabaudi orthologue, PcMSP1p19 (also called PcMEGF) [21, 30] were maintained in continuous culture on $\mathrm{O}^{+}$erythrocytes in RPMI supplemented with $0.5 \%$ Albumax and $1 \mu \mathrm{g} \mathrm{mL} \mathrm{m}^{-1}$ gentamycin, in candle jars [31]. Merozoites were collected as described previously [19] from cultures with greater than $5 \%$ parasitaemia after centrifugation $5 \mathrm{~min}$ at $400 \times g$, to remove red blood cells (RBCs), followed by a second centrifugation of the supernatant for $20 \mathrm{~min}$ at $1500 \times g$.

\section{PMN preparation}

PMNs were prepared as described previously [19]. Briefly, blood samples from six to seven healthy donors were collected into EDTA-K3 tubes, layered onto FicollHistopaque (density 1.077, Sigma) and centrifuged at RT for $30 \mathrm{~min}$ at $400 \times \mathrm{g}$. PMNs were harvested at the Ficoll$\mathrm{RBC}$ interface and residual RBCs were lysed by incubation in $8.32 \mathrm{~g} \mathrm{~L}^{-1} \mathrm{NH}_{4} \mathrm{Cl}, 0.8 \mathrm{~g} \mathrm{~L}^{-1}$ sodium bicarbonate, and $0.043 \mathrm{~g} \mathrm{~L}^{-1}$ EDTA for $8 \mathrm{~min}$ at $4{ }^{\circ} \mathrm{C}$. PMNs were washed twice with Hank's balanced salt solution (HBSS), enumerated using Trypan blue, and resuspended in PBS at $1-5 \times 10^{7}$ cells $\mathrm{mL}^{-1}$.

\section{Chemiluminescence monitoring and determination of standardized ADRB index}

Chemiluminescence was measured as described previously [19] using opaque 96-well plates (Berthold), and a MicroLumat Plus 96 luminometer (Berthold). Briefly, merozoite pellets $(40 \mu \mathrm{L})$ were incubated with $10 \mu \mathrm{L}$ of test or control sera for at least $30 \mathrm{~min}$ at $37^{\circ} \mathrm{C}$. PMN (100 $\mu \mathrm{L}$ at $1-5 \times 10^{7}$ cells $\left.\mathrm{mL}^{-1}\right)$ and isoluminol $(100 \mu \mathrm{L}$ of 
1:100 dilution in PBS of $4 \mathrm{mg} \mathrm{mL}^{-1}$ stock in DMSO) were loaded rapidly using an Eppendorf multipipette 4780. To facilitate rapid handling, only 40-50 wells per plate were used using the HIS as systematic internal control in the first and last wells. Plate reading started immediately, and continued for $1 \mathrm{~h}$.

Data are presented as standardized activity index of merozoite ADRB calculated as

$$
\begin{aligned}
\mathrm{ADRB} \text { index }= & (\text { rlu maximum sample } / \text { rlu maximum HIS }) \\
& \times 1000
\end{aligned}
$$

where rlu maximum HIS is an average of the first and last wells on the plate. Only experiments in which the rlu maximum HIS was $\geq 100$ ( $\geq 6 \times$ background), were included in the analyses. An additional internal control with the same positive serum was included in each run.

\section{Chemiluminescence assay using antigen coated on plates}

Baculovirus PfMSP1p19 was coated on white Nunc opaque Maxisorp plates (Dynatech) at $1 \mu \mathrm{g} / \mathrm{mL}$ overnight at $4{ }^{\circ} \mathrm{C}$. Plates were then washed three times with PBS-Tween- $0.05 \%$ and blocked for $1 \mathrm{~h}$ with PBS-BSA2 \% before a second wash. Native (undecomplemented) sera diluted 1:5 in PBS were then added and incubated for $1 \mathrm{~h}$ at $37^{\circ} \mathrm{C}$. PMN and isoluminol were then added as for classical ADRB (see above) $[19,22]$, before reading in the MicroLumat Plus 96 luminometer (Berthold).

\section{Statistical analysis of ADRB assay data}

ELISA and/or ADRB data were analysed for statistical significance using the Wilcoxon signed rank test and the Spearman rank correlation test for non-normally distributed data, and $P$ values $<0.05$ were considered significant. Multiple regression analysis including age of individuals, $\mathrm{Ab}$ response to PfMSP1p19 and ADRB was done using R software.

\section{Results}

Prevalence and levels of PfMSP1p19-binding IgG and their relationship with antibody-dependent respiratory burst activity in individual sera

The prevalence and levels of IgG specific for PfMSP1p19 were first determined in a set of 233 endemic sera, including 119 from Dielmo (holo-endemic) and 114 from Ndiop (meso-endemic). Antibody responses expressed as ELISA OD ratios are summarized in Table 1. Prevalence of IgG to PfMSP1p19 was high, with 74 and $86 \%$ of responders in the Dielmo and Ndiop cohorts, respectively. Both seropositivity and IgG levels to PfMSP1p19 were higher in Dielmo than Ndiop $(P=0.023$ and $P=0.009$ by Fisher's exact test and Mann Withney rank sum test, respectively).

ADRB and antibodies to PfMSP1p19 correlated with age. A quite significant correlation between the magnitude of ADRB index and IgG responses to PfMSP1p19 was found (Rho $=0.71$ and $0.68, P<10^{-3}$ for Dielmo and Ndiop, respectively). Stratification of antibody responses to PfMSP1p19 of individuals (Fig. 1) into non-responders (NR), positive responders (PR, individuals with OD-ratio $>2$ ) and high responders (HR; individuals with OD-ratio $>7$ ) outlined a significant association of ADRB with increasing response to PfMSP1p19 $\left(P<10^{-3}\right)$. The significant contribution of PfMSP1p19 antibody responses to ADRB was confirmed highly significant $P<10^{-4}$ in an age-adjusted linear regression model.

\section{Naturally acquired antibodies binding to PfMSP1p19 are major inducers of ADRB activity}

To demonstrate a role for PfMSP1p19-specific IgG in the ADRB, assays were carried out using either P. falciparum D10 (D10-PfM3') or transgenic D10 PcMEGF merozoites, in which PfMSP1p19 has been replaced by the noncross reactive $P$. chabaudi homologue, PcMSP1p19 [30], such that differences in observed chemiluminescence signals can be attributed to antibodies specific for PfMSP1p19 [21]. As shown in Fig. 2, comparative ADRBs were performed using 21 individual sera with high antiPfMSP1p19 ELISA OD-ratios. Chemiluminescence elicited by the transgenic PcMEGF merozoites was significantly reduced compared to D10-PfM3' for all individual

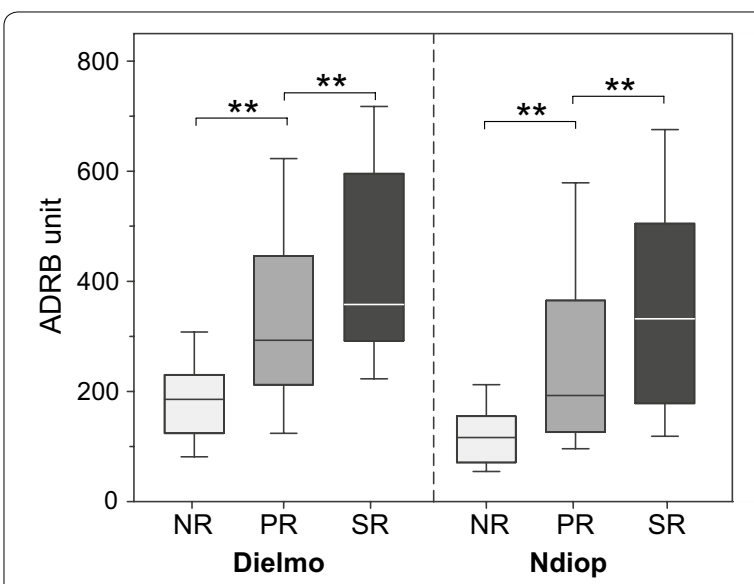

Fig. 1 ADRB responses stratified by anti PfMSP1 19 antibody levels in the two villages. ADRB measured in 119 and 114 sera from Dielmo and Ndiop, respectively are plotted as function of their anti-PfMSP1 1 19 antibodies responses stratified into three levels: non-responders (NR), positive responders ( $\mathrm{PR}, \mathrm{OD}$ ratio $>2$ ) and strong responders (SR, OD ratio > 7). The SR level was selected according to previous study [21] underlining this critical threshold of antibody levels to PfMSP1p19 in Ndiop. Boxplot graphs show that the profile of ADRB responses strongly and significantly increase $\left(P<10^{-3}\right)$ with increasing anti-PfMSP1P19 antibodies content. The median is indicated by a line, the $50 \%$ percentiles indicated by the box limits and the upper and lower $25 \%$ by whiskers 

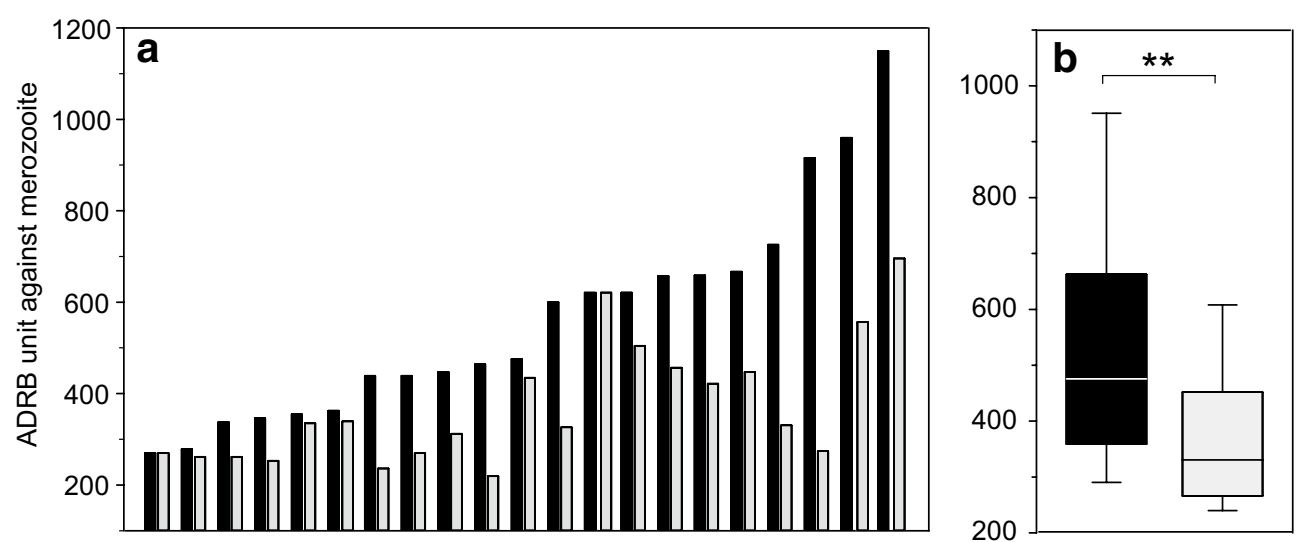

Fig. 2 Naturally-acquired anti-PfMSP1P19 antibodies are major inducers of ADRB activity against $P$. falciparum merozoites. a ADRB chemiluminescence readout for 21 sera with high anti-PfMSP1 1 19 OD-ratios tested using either P. falciparum D10 merozoites (D10-PfM3', black bars) or transgenic D10 merozoites in which the P. falciparum MSP1P19 gene was replaced by its P. chabaudi orthologue (D10-PcMEGF, black bars). D10-PfM3' and D10-PCMEGF merozoites were tested in the same plate, with the same polymorphonuclear cells batch. b Boxplot graph showing the significant $\left(\mathrm{P}<10^{-3}\right)$ overall distribution of ADRB generated using sera and D10-PfM3' or D10-PcMEGF transgenic merozoites. The median is indicated by a line, the $50 \%$ percentiles indicated by the box limits and the upper and lower $25 \%$ by whiskers

sera $\left(P<10^{-3}\right)$, varying from 7 to $54 \%$ inhibition, with a mean of $33.1 \%$. Importantly a similar reduction of chemiluminescence was observed using the HIS pool of 30 immune antisera, none of which was selected for presence of PfMSP1p19-binding antibodies (and thus unrelated to the 21 individually sera analysed above). These results indicate that antibodies induced by natural $P$. falciparum infection to this single conserved antigen represent a good proportion of antibodies mediating reactive oxygen species (ROS) release by neutrophils.

Impact of PfMSP1p19 antibody depletion on ADRB activity To explore contribution of PfMSP1p19-specific antibody to the ADRB readout, another approach was developed, involving depletion of specific antibodies by adsorption on the recombinant antigen. Efficiency of depletion monitored by ELISA on the antigen showed that the depletion protocol was highly effective. Median titre units decreased from 511 to 1 after anti-PfMSP1p19 depletion, indicating a depletion efficiency $>99 \%$.

Figure 3 summarizes the effect of anti-PfMSP1p19 antibody depletion on ADRB activity. A paired comparison of ADRB indexes before and after depletion of anti-PfMSP1p19 in 21 individual immune sera showed a $33.2 \%$ average reduction after depletion (range 0-70\%; $P=0.0017$ ), confirming the results obtained using the transgenic D10 PcMEGF merozoites.

\section{Solid-phase ADRB driven by PfMSP1p19 antigen and human sera}

To further explore the contribution of PfMSP1p19-specific antibody to $\mathrm{ADRB}$, solid-phase $\mathrm{ADRB}$ was carried out using plates coated with the recombinant PfMSP1p19 antigen as sole antigen in the assay. A set of 21 sera from Dielmo and Ndiop and four sera from hospitalized urban malaria with variable levels of anti-PfMSP1p19 IgG were simultaneously tested.

The kinetics of luminescence and ROS production was different than from the previously described ADRB assay using entire merozoites [19]. The peak of luminescence, measured using isoluminol occurred around $30 \mathrm{~min}$, i.e. later than the peak occurring within $5 \mathrm{~min}$ in the usual the standard assay (Additional file 1) [19, 22]. High levels of luminescence were observed (around 2-3000 rlu), the highest signal being produced by the immune IgG positive control. Background rlu from the commercial naive control pool of sera was around 300 and very low signals were observed with individual naive European sera (rlu < 40).

The individual levels of ADRB measured in the set of sera are shown Fig. 4. The solid-phase PfMSP1p19-specific ADRB index varied depending on the individual. Importantly, there was a positive and significant relationship between IgG to PfMSP1p19 and solid-phase ADRB measures, with a correlation coefficient rho $=0.64$ $\left(P<10^{-3}\right)$.

\section{Discussion}

It has been previously demonstrated that PMN from healthy donors produce a respiratory burst in response to merozoites and IgG1 and/or IgG3 antibodies in sera from individuals living in malaria-endemic areas [19]. It was further shown that the merozoite-triggered ADRB activity of sera from humans living in endemic areas correlated 

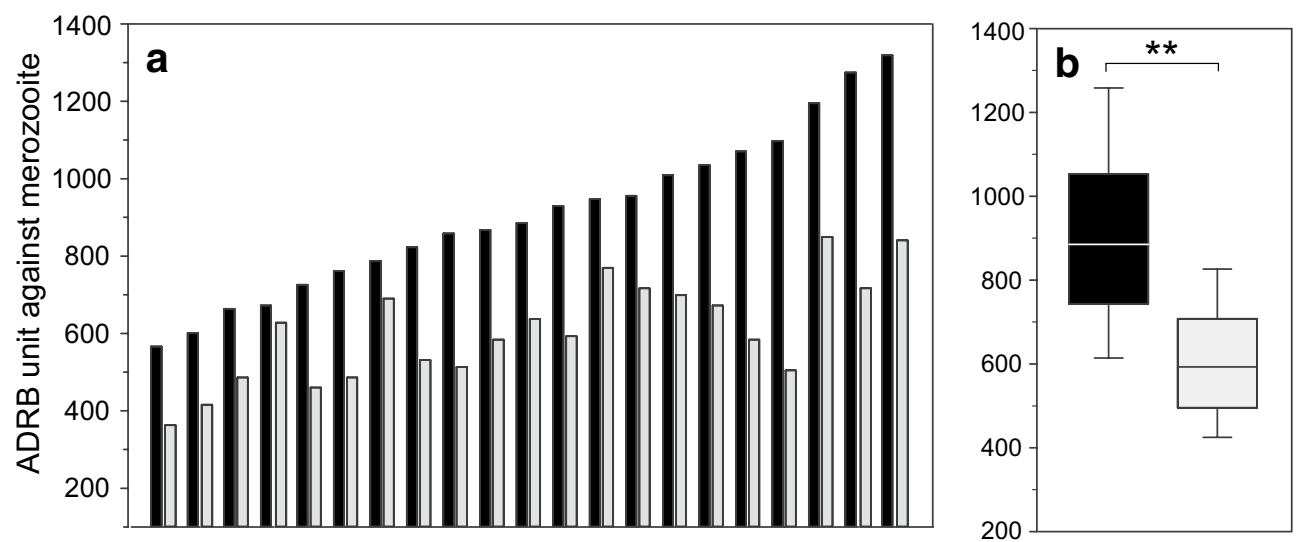

Fig. 3 Effect of anti-PfMSP1P19 depletion on ADRB activity against P. falciparum merozoites. A set of 21 sera were depleted from anti-PfMSP1 p19 antibodies using metal affinity immuno-adsorbent. a The ADRB level of each serum pair (undepleted and depleted, black and light bars, respectively) was plotted in increasing order of ADRB level of undepleted sera. $\mathbf{b}$ Boxplot graph showing the significant $\left(\mathrm{P}<10^{-3}\right)$ overall drop of $\mathrm{ADRB}$ from initial vs depleted sera. All sera selected had initial high levels of anti-PfMSP1 1919 antibodies. The median is indicated by a line, the $50 \%$ percentiles indicated by the box limits and the upper and lower $25 \%$ by whiskers

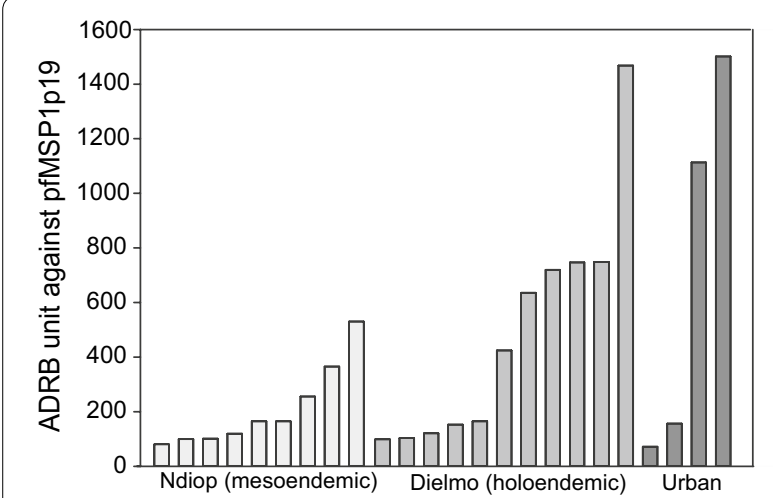

Fig. 4 ADRB activity of a set of 24 sera in the solid-phase assay using PFMSP1 p19-coated plates. Histograms of ADRB activity measured using PfMSP1 p19-coated in 9, 11 and 4 sera from Ndiop (light grey), Dielmo (medium grey) and urban malaria (dark grey), respectively, plotted in increasing order of ADRB units, respectively. The sera were chosen irrespective of their level of antibodies to PfMSP1 p19 antigen

with naturally acquired clinical protection from malaria. The work presented here identifies PfMSP1p19 as a target of antibodies mediating ADRB activity in sera from humans immunized by repeated exposure to P. falciparum infection. IgG responses to the recombinant PfMSP1p19 were highly correlated with ADRB activity in the two settings analysed. Importantly, depletion of sera from antibodies specific for PfMSP1p19 or use of merozoites expressing the non-cross-reactive rodent orthologue, PcMSP1p19 caused a significant reduction in the capacity of hyper-immune and high responder sera to mediate ADRB activity. Finally, ADRB could be triggered by incubating immune sera with recombinant PfMSP1p19 as sole target antigen.

The clinical implications of this work are two-fold. First, the results presented here strongly implicate human IgG antibodies' ability to react with the native PfMSP1p19 antigen displayed onto the merozoite surface as mediators of ADRB activity. Importantly, this target antigen exhibits limited antigenic diversity, an essential feature of vaccine candidates aiming to provide durable protection against polymorphic field parasite populations. Numerous sero-epidemiology studies have documented a high seroprevalence of antibodies to PfSMP1p19 [8, 9, 27, 32, 33]. The data observed here with two Senegalese cohorts living in different endemic conditions and moreover gathering different ethnic groups [34] are thus likely to be relevant for multiple endemic settings. Natural antibodies to PfMSP1p19 accounted for approximately $30 \%$ of the ADRB activity of immune human sera in this work. Interestingly, O'Donnell et al. [30] using immune sera from Papua New Guinea and the same isogenic parasite strains showed that antibodies to PfMSP1p19 were responsible for about $25 \%$ of the erythrocyte invasion inhibitory response of the human sera in vitro [30]. Thus these two assays provide a similar evaluation of the contribution of the anti-PfMSP1p19 specificity within the pool of antibodies reacting with merozoites. This indicates that indeed antibodies to PfMSP1p19 are a major component of the naturally-acquired, infection-elicited anti-merozoite response. A recent study conducted in the P. yoelii mouse model showed that vaccination with the AdHu5-PyMSP $1_{42}$ construct did not induce anti-PyMSP1p19 ADRB-triggering antibodies. Anti-PyMSP1p19 
antibodies contributing to ADRB were however observed in naïve as well as vaccinated mice after a primary infection, suggesting that infection elicits such antibodies [20].

Second, the data show that the baculovirus recombinant PfMSP1p19 recapitulates here the functional triggering activity of the merozoite-displayed native antigen. This is important information for vaccine development. Plasmodium MSP1p19 is composed of two intimately associated EGF domains, including 10-12 cysteines and five to six disulfide bonds, constituting an overall conformation not easily reproduced in lower order expression systems [35, 36], and human antibody recognition of PfMSP1p19 is totally conformation dependent [25, 37]. Moreover, evidence from the literature points to the potential importance of proper MSP1p19 conformation in evaluating the effectiveness of specific antibody responses. Anti-baculovirus PfMSP1p19 IgG levels in endemic sera from Dielmo and Ndiop were previously shown to correlate with both erythrocyte invasion inhibition by $P$. falciparum merozoites in vitro, and clinical protection from $P$. falciparum malaria in an age-adjusted multivariate analysis [9] (confirmed in two subsequent studies, R. Perraut and ML Varela, unpublished data). These results contrast with those of Roussilhon et al. [13] using sera from the same Senegalese sites, indicating no correlation of clinical protection with antibodies to a recombinant PfMSP1-19 construct corresponding to a single EGF domain with unlikely resemblance to native PfMSP1p19 [35, 36]. Similarly Dodoo et al. [32] found that IgG1 specific for baculovirus PfMSP1p19 was correlated with protection from clinical malaria in Ghanaian children, whereas a previous study in the same location using Escherichia coli GST-PfMSP1p19 fusion antigens showed no such association [38].

In the work reported here, focus was on IgG. However, ADRB was measured with immune sera, which might also contain specific IgA. The contribution of IgA to the observed responses remains to be investigated, as IgA has been shown to induce respiratory bursts to merozoites and MSP1-19 via Fc $\alpha$ R (CD89), which is constitutively expressed by neutrophils [18].

The association of antibodies to PfMSP1p19 with protection is incompletely captured using parameters such as antibody levels $[7,9,13,26,27,32,38,39]$ or GIA [8, 9]. The association with ADRB, itself associated with protection against clinical malaria, may somehow combine previous observations: high levels of anti-PfMSP1p19 antibodies that recognize the merozoite surface-displayed antigen may altogether inhibit invasion, efficiently trigger $\mathrm{ADRB}$ and possibly ADCI. These functional read-outs are not mutually exclusive, reflecting an association of antibodies with the native antigen on the merozoite surface. ADRB is rapid and as such stands out as a convenient assay for monitoring the anti-merozoite activity in human sera, and an interesting in vitro surrogate of clinical protection relevant for MSP-based vaccine candidates.

Here, three experimental approaches were used to study the contribution of specific antibodies to ADRB. Use of isogenic merozoites was appropriate for MSP1p19, because its function is apparently based entirely on its 2 EGF-domain structure, and can be brought about by an orthologous domain with differing antigenicity. This feature is unusual and may not apply to other antigens of interest [21]. Moreover, isogenic lines pairs expressing or not a specific antigen, are still rare. The data shown here validate the depletion approach and the solid-phase ADRB approach for the evaluation of other MSP vaccine candidates using the ADRB assay. The depletion protocol depended on using hexa-histidine-tagged antigens, which allowed the formation of antigen-antibody complexes in solution and subsequent removal by binding to the metallo-affinity resin, which was critical for efficient depletion. This methodology should be readily adaptable to antigens carrying other specific tags and presents the advantage of not requiring preparation of specific immuno-affinity chromatography reagents. The antigencoated ADRB proved a convenient test for functional screening of polyclonal sera for single antigen specificity such as for PfMSP1p19. However, the stimulation is rather qualitative, with a positive but not very strong correlation (65 \%) with level of antibodies measured by ELISA. The solid phase ADRB, where PMN activation is detected by the use of isoluminol, i.e., monitoring predominantly extracellular ROS, differs from the rapid peak response (within $5 \mathrm{~min}$ ) in standard ADRB using merozoites [19]. Kapeslki et al. reported that the peak of luminescence occurred later (20 min) when non-decomplemented plasma are used [22]. However, such a solid-phase test is easier of use than merozoite-based ADRB. Further improvement is required for optimization e.g. using IgG instead of sera, HBSS medium instead of PBS [40], luminol instead of isoluminol [22] and should conveniently complement the process of development for functional analysis targeting merozoite-derived vaccine candidates.

\section{Conclusions}

The results presented here show that antibodies to a single small conserved antigen, PfMSP1p19, are responsible for a good proportion of neutrophil ADRB, an activity that has been correlated with protection from clinical $P$. falciparum malaria in endemic areas. They provide further support for development of baculovirus recombinant PfMSP1p19 as a vaccine candidate. Importantly, the work opens the door to a new approach for the functional analysis of antibodies to other merozoite surface antigens, as a complement to the GIA and ADCI assays. 


\section{Additional file}

Additional file 1. Exemple of ADRB luminescence profiles recorded in the study using the standard ADRB assay (A) and the solid-phase PfMSP1p19-coated ADRB assay (B). In (B) the individual luminescence units (rlu) recorded using luminometer as function of time were measured in the reference HIS used as positive control in all assays (a), two PfMSP1p19positive individuals from Dielmo and Ndiop (b, c), one PfMSP1 1919-negative individuals from Dielmo (d), a pool of European sera used as negative control (e) and an individual European negative control ( $f$ ).

\section{Abbreviations}

ADCl: antibody dependent cellular inhibition; ADRB: antibody dependent respiratory burst; HIS: hyper-immune sera; MSP: merozoite surface protein; PMN: polymorphonuclear neutrophils; rlu: relative light units; ROS: reactive oxygen species; GIA: growth inhibition assay; NIS: non-immune sera; RT: room temperature; NR: non-responders; PR: positive responders; HR: high responders.

\section{Authors' contributions}

$\mathrm{RP}$ and OP designed and initiated the study. CJ and MLV conducted the laboratory experiments and statistical analyses, with help from AM, BM, AT and LM. Further, J-FT, CS, AT, LM, and BM supervised the collection of field samples and data. RP and OP drafted most of the paper, with important input from CJ and $M L V$, and comments from other authors. Figures and tables were prepared by $\mathrm{RP}, \mathrm{CJ}$ and MLV. All authors have read and approved the final manuscript.

\section{Author details}

${ }^{1}$ Unité d'Immunologie, Institut Pasteur de Dakar, Dakar, Senegal. ${ }^{2}$ Unité d'Immunologie Moléculaire des Parasites, Institut Pasteur, Paris, France.

${ }^{3}$ Unité d'Immunogénétique/UCAD, Institut Pasteur de Dakar, Dakar, Senegal.

${ }^{4}$ Unité d'Epidémiologie, Institut Pasteur de Dakar, Dakar, Senegal. ${ }^{5}$ Institut de Recherche pour le Développement (IRD), URMITE, Dakar, Senegal.

\section{Acknowledgements}

We are particularly grateful to Dr S Longacre who substantially contributed to the design and the execution of this work and generously provided the PfMSP1p19 antigen as well as to Prof B Crabb who kindly provided the isogenic P. falciparum D10 lines. We thank Prs A Dieye, L Diakhate and Dr M Delepierre and O Niass for helpful discussions and G Milon for reviewing the manuscript. S Rosario prepared the purified PfMSP1p19 antigen. We are grateful to the villagers from Ndiop and Dielmo who participated in this study. The work was supported by grants from the Institut Pasteur Foundation, the French Ministère de la Recherche et de la Technologie (Programme VIHPAL), the Fonds dédiés programme: Combatting Parasitic Diseases (Sanofi Aventis/ Ministère de l'Enseignement Supérieur et de la Recherche) and the 7th European Framework Programme, FP7/2007-2013, contract 242095, Evimalar. CJ received financial support from Pharmaciens Sans Frontières-Comité International and the Agence Universitaire de la Francophonie.

\section{Competing interests}

The authors declare that they have no competing interests.

Received: 15 April 2015 Accepted: 27 August 2015

Published online: 15 October 2015

\section{References}

1. WHO. World malaria report 2013. Geneva: World Health Organization; 2014. http://www.whoint/malaria/publications/ world_malaria_report_2013/.

2. Dondorp AM, Nosten F, Yi P, Das D, Phyo AP, Tarning J, et al. Artemisinin resistance in Plasmodium falciparum malaria. N Engl J Med. 2009;361:455-67.

3. Tun KM, Imwong M, Lwin KM, Win AA, Hlaing TM, Hlaing T, Lin K, et al. Spread of artemisinin-resistant Plasmodium falciparum in Myanmar: a cross-sectional survey of the K13 molecular marker. Lancet Infect Dis. 2015;15:415-21.

4. Ballou WR, Arevalo-Herrera M, Carucci D, Richie TL, Corradin G, Diggs C, et al. Update on the clinical development of candidate malaria vaccines. Am J Trop Med Hyg. 2004;71:239-47.

5. Girard MP, Reed ZH, Friede M, Kieny MP. A review of human vaccine research and development: malaria. Vaccine. 2007;25:1567-80.

6. Cowman AF, Crabb BS. Invasion of red blood cells by malaria parasites. Cell. 2006;124:755-66.

7. Fowkes FJ, Richards JS, Simpson JA, Beeson JG. The relationship between anti-merozoite antibodies and incidence of Plasmodium falciparum malaria: a systematic review and meta-analysis. PLoS Med. 2010;7:e1000218.

8. John CC, O'Donnell RA, Sumba PO, Moormann AM, de Koning-Ward TF, et al. Evidence that invasion-inhibitory antibodies specific for the 19-kDa fragment of merozoite surface protein-1 (MSP-1 19) can play a protective role against blood-stage Plasmodium falciparum infection in individuals in a malaria endemic area of Africa. J Immunol. 2004;173:666-72.

9. Perraut R, Marrama L, Diouf B, Sokhna C, Tall A, Nabeth P, Trape JF, et al. Antibodies to the conserved C-terminal domain of the Plasmodium falciparum merozoite surface protein 1 and to the merozoite extract and their relationship with in vitro inhibitory antibodies and protection against clinical malaria in a Senegalese village. J Infect Dis. 2005;191:264-71.

10. Singh S, Miura K, Zhou H, Muratova O, Keegan B, Miles A, et al. Immunity to recombinant Plasmodium falciparum merozoite surface protein 1 (MSP1): protection in Aotus nancymai monkeys strongly correlates with anti-MSP1 antibody titer and in vitro parasite-inhibitory activity. Infect Immun. 2006;74:4573-80.

11. Aribot G, Rogier C, Sarthou JL, Trape JF, Balde AT, Druilhe P, et al. Pattern of immunoglobulin isotype response to Plasmodium falciparum bloodstage antigens in individuals living in a holoendemic area of Senegal (Dielmo, west Africa). Am J Trop Med Hyg. 1996;54:449-57.

12. Garraud O, Mahanty S, Perraut R. Malaria-specific antibody subclasses in immune individuals: a key source of information for vaccine design. Trends Immunol. 2003:24:30-5.

13. Roussilhon C, Oeuvray C, Muller-Graf C, Tall A, Rogier C, Trape JF, et al. Long-term clinical protection from falciparum malaria is strongly associated with lgG3 antibodies to merozoite surface protein 3. PLoS Med. 2007:4:e320.

14. Bouharoun-Tayoun H, Oeuvray C, Lunel F, Druilhe P. Mechanisms underlying the monocyte-mediated antibody-dependent killing of Plasmodium falciparum asexual blood stages. J Exp Med. 1995;182:409-18.

15. Kumaratilake LM, Ferrante A, Jaeger T, Rzepczyk CM. Effects of cytokines, complement, and antibody on the neutrophil respiratory burst and phagocytic response to Plasmodium falciparum merozoites. Infect Immun. 1992;60:3731-8.

16. Salmon D, Vilde JL, Andrieu B, Simonovic R, Lebras J. Role of immune serum and complement in stimulation of the metabolic burst of human neutrophils by Plasmodium falciparum. Infect Immun. 1986;51:801-6.

17. Pleass RJ, Ogun SA, McGuinness DH, van de Winkel JG, Holder AA, Woof JM. Novel antimalarial antibodies highlight the importance of the antibody Fc region in mediating protection. Blood. 2003;102:4424-30.

18. Shi J, McIntosh RS, Adame-Gallegos J, Dehal PK, van Egmond M, van de Winkel J, et al. The generation and evaluation of recombinant human IgA specific for Plasmodium falciparum merozoite surface protein 1-19 (PfMSP1 19). BMC Biotechnol. 2011;11:77.

19. Joos C, Marrama L, Polson HE, Corre S, Diatta AM, Diouf B, et al. Clinica protection from falciparum malaria correlates with neutrophil respiratory bursts induced by merozoites opsonized with human serum antibodies. PLoS One. 2010;5:e9871.

20. Llewellyn D, de Cassan SC, Williams AR, Douglas AD, Forbes EK, AdameGallegos JR, et al. Assessment of antibody-dependent respiratory burst activity from mouse neutrophils on Plasmodium yoelii malaria challenge outcome. J Leukoc Biol. 2014;95:369-82.

21. O'Donnell RA, Saul A, Cowman AF, Crabb BS. Functional conservation of the malaria vaccine antigen MSP-119 across distantly related Plasmodium species. Nat Med. 2000;6:91-5

22. Kapelski S, Klockenbring T, Fischer R, Barth S, Fendel R. Assessment of the neutrophilic antibody-dependent respiratory burst (ADRB) response to Plasmodium falciparum. J Leukoc Biol. 2014;96:1131-42. 
23. Trape JF, Pison G, Spiegel A, Enel C, Rogier C. Combating malaria in Africa. Trends Parasitol. 2002;18:224-30.

24. Trape JF, Rogier C, Konate L, Diagne N, Bouganali H, Canque B, Legros F, Badji A, Ndiaye G, Ndiaye P, et al. The Dielmo project: a longitudinal study of natural malaria infection and the mechanisms of protective immunity in a community living in a holoendemic area of Senegal. Am J Trop Med Hyg. 1994;51:123-37.

25. Bonnet S, Petres S, Holm I, Fontaine T, Rosario S, Roth C, Longacre S. Soluble and glyco-lipid modified baculovirus Plasmodium falciparum C-terminal merozoite surface protein 1, two forms of a leading malaria vaccine candidate. Vaccine. 2006;24:5997-6008.

26. Perraut R, Marrama L, Diouf B, Fontenille D, Tall A, Sokhna C, Trape JF, Garraud O, Mercereau-Puijalon O. Distinct surrogate markers for protection against Plasmodium falciparum infection and clinical malaria identified in a Senegalese community after radical drug cure. J Infect Dis. 2003;188:1940-50.

27. Perraut R, Richard V, Varela ML, Trape JF, Guillotte M, Tall A, et al. Comparative analysis of IgG responses to Plasmodium falciparum MSP1p19 and PF13-DBL1alpha1 using ELISA and a magnetic bead-based duplex assay (MAGPIX(R)-Luminex) in a Senegalese meso-endemic community. Malar J. 2014;13:410.

28. Belard S, Issifou S, Hounkpatin AB, Schaumburg F, Ngoa UA, Esen M, et al. $A$ randomized controlled phase $\mathrm{Ib}$ trial of the malaria vaccine candidate GMZ2 in African children. PLoS One. 2011;6:e22525.

29. Miura K, Orcutt AC, Muratova OV, Miller LH, Saul A, Long CA. Development and characterization of a standardized ELISA including a reference serum on each plate to detect antibodies induced by experimental malaria vaccines. Vaccine. 2008;26:193-200

30. O'Donnell RA, de Koning-Ward TF, Burt RA, Bockarie M, Reeder JC, Cowman AF, et al. Antibodies against merozoite surface protein (MSP)-1(19) are a major component of the invasion-inhibitory response in individuals immune to malaria. J Exp Med. 2001;193:1403-12.

31. Diouf B, Pradines B, Spiegel A, Trape JF, Dieye A, Parzy D, et al. Re-assessment of culture inhibition assays and reinvasion of $P$. falciparum for the appraisal of immunity of individuals living in an endemic area (in French). Dakar Med. 2002;47:5-11.
32. Dodoo D, Aikins A, Kusi KA, Lamptey H, Remarque E, Milligan P, et al. Cohort study of the association of antibody levels to AMA1, MSP119, MSP3 and GLURP with protection from clinical malaria in Ghanaian children. Malar J. 2008;7:142

33. Torres KJ, Clark EH, Hernandez JN, Soto-Cornejo KE, Gamboa D, Branch $\mathrm{OH}$. Antibody response dynamics to the Plasmodium falciparum conserved vaccine candidate antigen, merozoite surface proteinC-terminal 19kD (MSP1-19kD), in Peruvians exposed to hypoendemic malaria transmission. Malar J. 2008;7:173.

34. Sakuntabhai A, Ndiaye R, Casademont I, Peerapittayamongkol C, Rogier C, Tortevoye P, et al. Genetic determination and linkage mapping of Plasmodium falciparum malaria related traits in Senegal. PLoS One. 2008;3:e2000.

35. Chitarra V, Holm I, Bentley GA, Petres S, Longacre S. The crystal structure of C-terminal merozoite surface protein 1 at $1.8 \mathrm{~A}$ resolution, a highly protective malaria vaccine candidate. Mol Cell. 1999;3:457-64.

36. Pizarro JC, Chitarra V, Verger D, Holm I, Petres S, Dartevelle S, et al. Crystal structure of a Fab complex formed with PfMSP1-19, the C-terminal fragment of merozoite surface protein 1 from Plasmodium falciparum: a malaria vaccine candidate. J Mol Biol. 2003;328:1091-103.

37. Holm I, Nato F, Mendis KN, Longacre S. Characterization of C-terminal merozoite surface protein-1 baculovirus recombinant proteins from Plasmodium vivax and Plasmodium cynomolgi as recognized by the natural anti-parasite immune response. Mol Biochem Parasitol. 1997;89:313-9.

38. Dodoo D, Theander TG, Kurtzhals JA, Koram K, Riley E, Akanmori BD, et al. Levels of antibody to conserved parts of Plasmodium falciparum merozoite surface protein 1 in Ghanaian children are not associated with protection from clinical malaria. Infect Immun. 1999;67:2131-7.

39. Arnot DE, Cavanagh DR, Remarque EJ, Creasey AM, Sowa MP, Morgan WD, et al. Comparative testing of six antigen-based malaria vaccine candidates directed toward merozoite-stage Plasmodium falciparum. Clin Vaccine Immunol CVI. 2008;15:1345-55.

40. Freitas M, Porto G, Lima JL, Fernandes E. Optimization of experimental settings for the analysis of human neutrophils oxidative burst in vitro. Talanta. 2009;78:1476-83.

\section{Submit your next manuscript to BioMed Central and take full advantage of:}

- Convenient online submission

- Thorough peer review

- No space constraints or color figure charges

- Immediate publication on acceptance

- Inclusion in PubMed, CAS, Scopus and Google Scholar

- Research which is freely available for redistribution

Submit your manuscript at

www.biomedcentral.com/submit

C Biomed Central 